\title{
An Injectable Composite Gelatin Hydrogel with pH Response Properties
}

\author{
Baoguo Chen ${ }^{1}$ and Xiaohong $\mathrm{Hu}^{2}$ \\ ${ }^{1}$ Research Center for Science Technology and Society, Fuzhou University of International Studies and Trade, Fuzhou 350202, China \\ ${ }^{2}$ School of Material Engineering, Jinling Institute of Technology, Nanjing 211169, China \\ Correspondence should be addressed to Xiaohong Hu; hxh@jit.edu.cn
}

Received 26 August 2016; Accepted 22 November 2016; Published 11 January 2017

Academic Editor: Silvia Licoccia

Copyright (c) 2017 B. Chen and X. Hu. This is an open access article distributed under the Creative Commons Attribution License, which permits unrestricted use, distribution, and reproduction in any medium, provided the original work is properly cited.

\begin{abstract}
On account of minimally invasive procedure and of filling irregular defects of tissues, injectable hydrogels are increasingly attractive in biomedical fields. However, traditional hydrogel formed by simple physical interaction or in situ crosslinking had inevitably some drawbacks such as low mechanical strength and lack of multifunctional properties. Though many investigations had successfully modified traditional injectable hydrogel to obtain both mechanical and functional properties, an acetalated $\beta$-cyclodextrin (Ac$\beta$-CD) nanoparticle composite injectable hydrogel designed in the research was another effective and efficient choice to solve the drawbacks. First of all, gelatin derivative (G-AA) and Ac- $\beta-C D$ were synthesized to prepare hydrogel and nanoparticle, respectively. In order to ensure good compatibility between nanoparticle and macromonomer and provide crosslink points between nanoparticle and macromonomer, G-AA was simultaneously functionalized onto the surface of Ac- $\beta$-CD nanoparticle during the fabrication of Ac- $\beta$-CD nanoparticle using one-step method. Finally, injectable composite hydrogel was obtained by photoinitiated polymerization in situ. Hydrogel properties like gelation time and swelling ratio were investigated. The viscoelastic behavior of hydrogels confirmed that typical characteristics of crosslinked elastomer for all hydrogel and nanoparticle in hydrogel could improve the mechanical property of hydrogel. Moreover, the transparency with time had verified obvious acid-response properties of hydrogels.
\end{abstract}

\section{Introduction}

On account of minimally invasive procedure and of filling irregular defects of tissues, injectable hydrogels are increasingly attractive in biomedical fields $[1,2]$. Generally, the properties of biocompatibility, biodegradability, mild solidification conditions, and nontoxic initiating system were fundamental requisites for biomaterials including injectable hydrogels $[1,2]$. As for hydrogels, their highly hydrated structure can mimic the natural extracellular matrix (ECM) of body and lead to the ease of exchange of gas, nutrients, and metabolite substances [3]. Besides biocompatibility, natural polymers like polypeptide and polysaccharide have innate biodegradability, which gives these materials suitable characteristics for injectable hydrogels [4]. To date, many natural macromolecules such as collagen, gelatin, chitosan, alginate, and hyaluronic acid (HA) are fabricated into the injectable hydrogels [5-11]. Among these materials, gelatin is a hydrolysis product of collagen and contains bioactive sequences, which lead to its widespread applications in tissue engineering and drug delivery $[5,6,8]$. Despite these merits, injectable gelatin cannot be formed by only interactions between molecules or simple crosslinking, which is also a common drawback of natural polymers. For this reason, gelatin was modified with reactive functional groups like vinyl groups, acetenyl groups, and thiol, and injectable gelatin hydrogel was fabricated by photoinitiated polymerization or click chemistry in previous research. In the research, gelatin was functionalized with acrylic acid (AA) and photoinitiated polymerization was used to fabricate injectable hydrogel in view of its effectivity and efficiency.

However, traditional hydrogels have also some drawbacks like low mechanical properties and multiresponse deficiency due to unicity of polymer network [9]. Another injectable carrier (particles with a size ranging from nanometer to micrometer) possesses flexible properties due to adjustable structure, 
which is just able to compensate for drawbacks of hydrogel [12-15]. Therefore, combining hydrogel with particles is an optimized method to fabricate a novel injectable material. Recently, an injectable scaffold of poly(lactic-co-glycolic acid) microparticles/chitosan hydrogel and a $\mathrm{pH}$-sensitive injectable nanoparticle composite hydrogel was prepared for tissue engineering and anticancer drug delivery application $[9,16]$. The previous research work has proven that composite hydrogels had better properties when nanoparticle could be crosslinked or have strong interaction with hydrogel in the process of hydrogel formation $[8,9,16]$.

Among the applied particles, low $\mathrm{pH}$-triggered particle attracts increasing interest based on the fact that $\mathrm{pH}$ value in infectious and inflammatory sites and in tumor tissue exhibits weak acid environment [17]. Synthesized ester polymers or copolymers could degrade in acid environment, which makes them be primary materials for low $\mathrm{pH}$-triggered nanoparticles $[15,18]$. But the biocompatibility of either synthesized ester polymer or its degraded product is not ideal for biomedical application. The recent emerging of acetalated cyclodextrins (Ac-CDs) changes the situation [17, 19-22]. Ac$\mathrm{CD}$ could be prepared to be low $\mathrm{pH}$-triggered nanoparticles by microemulsion method under the stability of emulgator $[17,19-22]$. In view of the strong interaction between hydrogel network and Ac-CD nanoparticles, double carbon functional Ac-CD nanoparticles were needed for the fabrication of injectable composite gelatin hydrogel. Considering the amphiphilic property of gelatin and the fact from previous report that emulgator was inevitably absorbed on the surface of nanoparticle stably in the process of nanoparticle formation through microemulsion method, we use abovementioned AA functionalized gelatin (G-AA) as an emulgator to prepare Ac- $\beta$-CD nanoparticles in the research. Finally, an injectable composite gelatin hydrogel was obtained by compounding of the hydrogel with Ac- $\beta$-CD nanoparticles, aiming at providing $\mathrm{pH}$ response properties for hydrogel and improving the hydrogel mechanical strength.

\section{Experimental Section}

2.1. Materials. $\beta$-Cyclodextrin ( $\beta$-CD), 2-methoxypropene, pyridinium 4-toluene sulfonate, dimethyl sulfoxide (DMSO), dichloromethane (DCM), and gelatin were obtained from Shanghai Chemical Industries Co., Ltd. (China). 1-Ethyl-3-(3dimethylaminopropyl) carbodiimide hydrochloride (EDC) and N-hydroxysuccinimide (NHS) were obtained from Sigma. 2-Hydroxy-1-[4-(hydroxyethoxy)phenyl]-2-methyl-1propanone (Irgacure 2959) was obtained from Ciba Specialty Chemicals. Acrylic acid (AA) was purified via distillation under reduced pressure. All other chemicals and reagents were used as received.

2.2. Synthesis of $G-A A$ and $A c-\beta-C D$. Acrylic acid modified gelatin (G-AA) was obtained by amidation under catalyzation of EDC. Briefly, $500 \mathrm{mg}$ EDC and $200 \mathrm{mg}$ NHS were successively added to $1.6 \%$ AA solution with $\mathrm{pH}$ value of 4.0-5.0 and adjusted by $\mathrm{NaOH}$ to activate carboxyl group. After $30 \mathrm{~min}, 50 \mathrm{~mL} 2 \%$ gelatin solution was added to the mixture to continue the reaction for the following $10 \mathrm{~h}$ at room temperature. Finally, G-AA was obtained by dialyzing of the resulted mixture and freeze-drying.

Acetalated $\beta$-cyclodextrin (Ac- $\beta$-CD) was synthesized by acetalization. Briefly, $50 \mathrm{mM} \beta-\mathrm{CD}$ and $5 \mathrm{mM}$ pyridinium $\mathrm{p}$ toluene sulfonate were orderly dissolved in anhydrous DMSO under nitrogen atmosphere, into which 40 molar times of 2methoxypropene were dropped. After the reaction had been conducted at $30^{\circ} \mathrm{C}$ for $6 \mathrm{~h}$, Ac- $\beta$-CD was precipitated from basic water, collected by filtration, and lastly lyophilized to a white powder.

The products were characterized by nuclear magnetic resonance hydrogen spectrum $\left({ }_{1} \mathrm{H}\right.$ NMR, BRUKER AV500).

2.3. Fabrication of Ac- $\beta-C D$ Nanoparticle. Ac- $\beta-C D$ nanoparticles were prepared by microemulsion evaporation method using G-AA as multifunctional emulsifier. Briefly, $1 \mathrm{~mL}$ of $10 \% \mathrm{w} / \mathrm{v}$ Ac- $\beta$-CD/DCM solution was emulsified via probe sonication (Scientz, JY92-II) into $6 \mathrm{~mL}$ of $3 \%$ w/v G-AA aqueous solution. The obtained emulsion was immediately added to $20 \mathrm{~mL}$ of $1 \% \mathrm{w} / \mathrm{v}$ G-AA solution to evaporate DCM under magnetic stirring. Finally, Ac- $\beta-C D$ nanoparticles were collected by centrifugation $(14000 \mathrm{rpm}$, $10 \mathrm{~min}$ ) after $10 \mathrm{~h}$. The collected nanoparticles were washed several times with basic water, lyophilized, and characterized by Fourier-transformed infrared spectroscopy (FTIR, Nicolet IS10) and scanning electron microscope (SEM, S8100). The solubility of nanoparticle was characterized by transparency using UV spectroscopy (Cary 50).

2.4. Preparation of Injectable Composite Hydrogel. The collected nanoparticles were suspended with $15 \%$ (w/v) G-AA aqueous solution to obtain homogeneous suspension, to which Irgacure 2959 (photoinitiator) with final concentration of $0.05 \% \mathrm{w} / \mathrm{v}$ was added and incubated at $37^{\circ} \mathrm{C}$ for $5 \mathrm{~min} .200$ $\mu \mathrm{L}$ of the mixtures was injected into circle models, covered with coverslip, and irradiated by $365 \mathrm{~nm}$ UV light with a power of $\sim 10 \mathrm{~mW} / \mathrm{cm}^{2}$. Gelation time was recorded when the solution lost its fluidity.

2.5. Characterization of Injectable Composite Hydrogel. After being irradiated for $30 \mathrm{~min}$, hydrogels were obtained, freezedried, and weighed $\left(W_{0}\right)$. The dried hydrogel was hydrated in water at $37^{\circ} \mathrm{C}$ for $24 \mathrm{~h}$. Then the equilibrium hydrated hydrogels were weighed $\left(W_{1}\right)$. The swelling ratio of the hydrogel is defined as $W_{1} / W_{0}$.

For the rheological properties test, hydrogels were prepared using another circular and transparent glass mold (25 mm diameter, $0.22 \mathrm{~mm}$ height), setting irradiation time of $30 \mathrm{~min}$. The samples were characterized by rheological measurement in a parallel plate mode using a strain-controlled rheometer (MCR102). Dynamic oscillatory mode (compression mode) was used to measure the storage and loss moduli. All tests were performed at $37^{\circ} \mathrm{C}$ with a fixed strain of $1 \%$.

In addition, the solubility or degradation of nanoparticle in hydrogel was characterized by transparency using UV spectroscopy (Cary 50) at $400 \mathrm{~nm}$.

2.6. Statistical Analysis. Data were analyzed using $t$-test for differences. Results are reported as means \pm standard deviation. The significant level was set at $p<0.05$. 


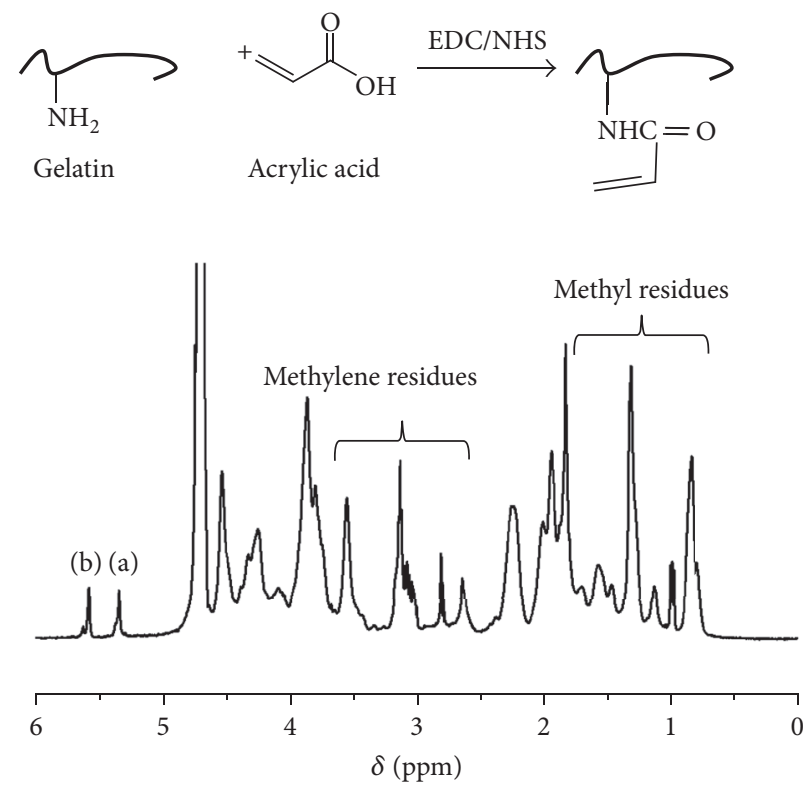

(a)

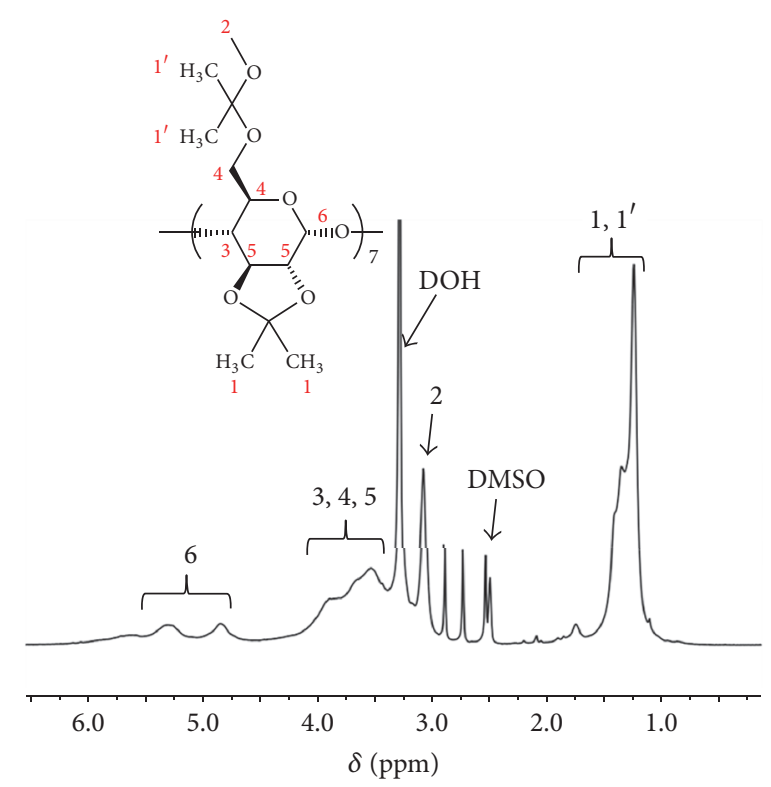

(b)

Figure 1: ${ }_{1} \mathrm{H}$ NMR spectra of (a) G-AA and (b) Ac- $\beta$-CD.

\section{Results}

3.1. Synthesis of $G-A A$ and $A c-\beta-C D$. The ${ }^{1}$ HNMR spectra were used for the structural information of G-AA and Ac$\beta$-CD in Figure 1. Firstly, in the ${ }^{1}$ HNMR spectrum of G-AA (Figure 1(a)), the chemical shifts from $0.6 \mathrm{ppm}$ to $1.7 \mathrm{ppm}$ belong to the protons of methyl residues and the chemical shifts from $2.5 \mathrm{ppm}$ to $3.7 \mathrm{ppm}$ are assigned to the protons of methylene residues, while $\delta=5.6$ (a) and $\delta=5.4$ (b) are typical chemical shifts belonging to the protons of $\underline{\mathrm{H}}_{2} \mathrm{C}=\mathrm{C}-$, which confirms the successful modification of gelatin by AA.

Secondly, the ${ }_{1} \mathrm{H}$ NMR spectrum of Ac- $\beta$-CD (Figure $1(\mathrm{~b}))$ is analyzed as follows: the chemical shifts at $3,4,5$, and 6 positions are, respectively, attributed to the protons of pyranose ring, constructed units of $\beta-C D$, the chemical shifts from $1.0 \mathrm{ppm}$ to $2.0 \mathrm{ppm}$ belong to the protons of $\mathrm{CH}_{3}-\mathrm{C}$ at 1 and $1^{\prime}$ positions, and $\delta=3.0 \mathrm{ppm}$ is assigned to the protons of $\mathrm{CH}_{3}-\mathrm{O}$ at 2 position. From the above analysis, the emergence of chemical shifts at $1,1^{\prime}$, and 2 positions verified acetalated groups on $\beta$-CD molecules. Further, since the number of protons is directly proportional to its relative average area, the degree of substituent (DS) can be quantified according to the relative average area of protons. Given the fact that protons at 3, 4, 5, and 6 positions were fixed, the DS of Ac$\beta$-CD was calculated by average area of $1,1^{\prime}$, and 2 positions to that of $3,4,5$, and 6 positions, which was $61 \%$. Ac- $\beta$-CD could dissolve in acid solution $(\mathrm{pH}<5.5)$, indicating the $\mathrm{pH}$ response property of the $\beta$-CD derivative.

3.2. Fabrication of Ac- $\beta-C D$ Nanoparticle. Figure 2(a) illustrated the fabrication process of Ac- $\beta$-CD nanoparticle through single oil-in-water (o/w) emulsion technique. Similar to traditional emulsion technique, nanoparticle was obtained after solvent had been volatilized. But, differently, crosslinkable gelatin (G-AA) was used as an emulsifier in the research. The formed nanoparticle exhibited nearly spherical morphology with slight coarse surface, confirmed by SEM image (Figure 2(b)). Further, the diameter of nanoparticle was $238 \pm 61 \mathrm{~nm}$ by statistics form SEM images. Moreover, chemical information of Ac- $\beta$-CD nanoparticle surface was characterized by FTIR spectrum (Figure 2(c)). At the same time, FTIR spectrum of Ac- $\beta$-CD derivative was also detected as a control sample in Figure 2(c). By comparing two spectra, a large amount of change including the obvious shift of absorbance bands from $3433 \mathrm{~cm}^{-1}$ to $3320 \mathrm{~cm}^{-1}$, the appearance of absorbance bands at $1446 \mathrm{~cm}^{-1}$, and the obvious enhance of peak at $1640 \mathrm{~cm}^{-1}$ and $1560 \mathrm{~cm}^{-1}$ emerged in the FTIR spectrum of nanoparticle. Analytically, $3320 \mathrm{~cm}^{-1}$ belonged to amide and amino groups, $1446 \mathrm{~cm}^{-1}$ is assigned to carboxylic acid peak, and $1640 \mathrm{~cm}^{-1}$ and $1560 \mathrm{~cm}^{-1}$ are attributed to amide I and amide II characteristic absorption. These differences confirmed the existence of G-AA (typical protein structure) on the surface of nanoparticle.

3.3. Preparation of Injectable Composite Hydrogel. The schematic illustration of injectable composite hydrogel fabrication was shown in Figure 3(a). It was found that Ac- $\beta-C D$ nanoparticles were compounded with the G-AA solution to obtain stable homogenous solution, which was ascribed to good compatibility between nanoparticle surface and macromonomer (G-AA). In the next step, the mixture was initiated by $\mathrm{I} 2959$ to form composite hydrogels. Here 4 types of composite hydrogel were prepared: pure hydrogel without nanoparticle (hydrogel0), composite hydrogel with $2 \mathrm{mg} / \mathrm{mL}$ Ac- $\beta$-CD nanoparticles (hydrogel1), composite hydrogel with 


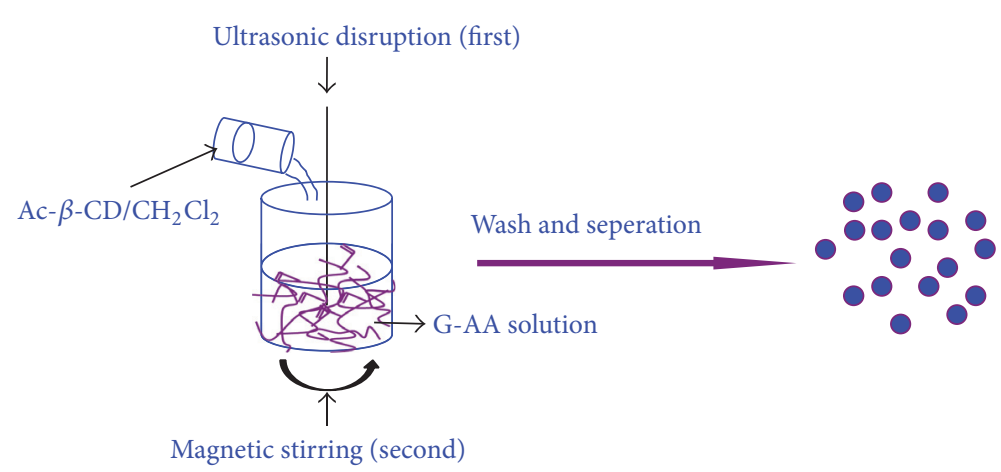

(a)

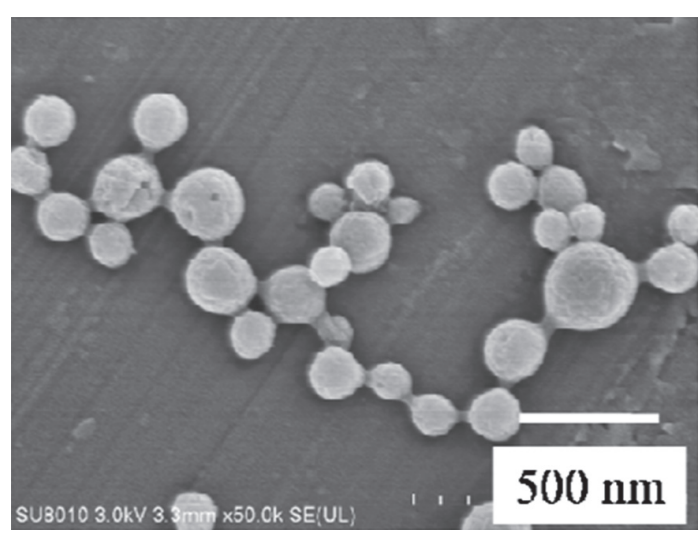

(b)

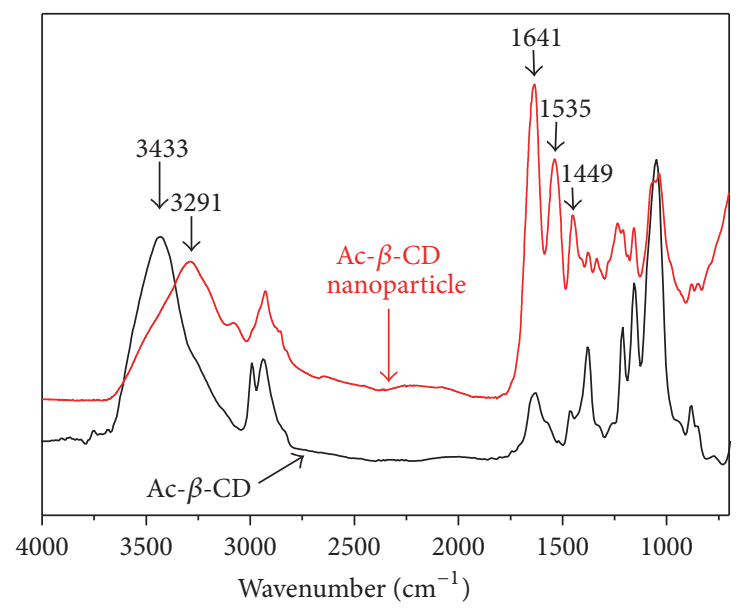

(c)

FIGURE 2: (a) Schematic illustration to show the formation of nanoparticle. (b) SEM image of Ac- $\beta$-CD nanoparticles. (c) FTIR spectra of Ac- $\beta$-CD and its nanoparticle.

$4 \mathrm{mg} / \mathrm{mL}$ Ac- $\beta$-CD nanoparticles (hydrogel2), and composite hydrogel with $8 \mathrm{mg} / \mathrm{mL}$ Ac- $\beta$-CD nanoparticles (hydrogel3). As an important evaluation indicator for injectable hydrogel, gelation time of the composite hydrogel was first studied in Figure 3(b). The gelation time showed slight increase with the increase of nanoparticle concentration until nanoparticle's concentration reached $4 \mathrm{mg} / \mathrm{mL}$ but without significant difference. Gelation of the G-AA and G-AA composite system, regardless of nanoparticles' concentration, is triggered mainly by crosslinking between the G-AA chains, which makes the solution lose its fluidity upon gelation. Nanoparticles might play a little inhibition for photoinitiated crosslinking reaction but not obvious. Swelling property can reflect the inner structure of hydrogel and influence substance exchange between hydrogels inside and outside environment. Thus swelling ratio of composite hydrogels was characterized in Figure 3(b) which was gradually decreased along with increase of the nanoparticles' concentration but without significant difference. Since the nanoparticle is not waterswelling particle, the mixed nanoparticles contribute only to the dried weight of the composites. Consequently, "apparent" swelling ratio of composite hydrogel showed a slight declined trend with nanoparticles' concentration.
The viscoelastic behavior of hydrogels was investigated in Figure 4. As a whole, storage moduli (Figure 4(a)) of all hydrogels were $1 \times 10^{3}-5 \times 10^{3} \mathrm{~Pa}$, which was 25-100 times higher than loss moduli (Figure 4(b)) of 10-200 Pa over the frequency range of $10^{-1}-10^{2} \mathrm{rad} / \mathrm{s}$. The result indicated that all hydrogels had some characteristics of elastomer. With increasing angular frequency, storage moduli showed little change and loss moduli varied gently with no sign of breakage as far as the measured angular frequency range was concerned. Theoretically, for uncrosslinked polymers, polymer chains do not have enough time to relax at high frequencies. Thus the elastic energy has been stored in polymer, which results in a high modulus of polymer. Nevertheless, when the polymer is crosslinked, chain relaxing and chain slipping are inhibited, resulting in relative stable modulus in the measured frequency range. Therefore, it was inferred that all hydrogels had a crosslinked elastomer structure. In further details, along with the increase of nanoparticle concentration, storage moduli were augmented correspondingly, while loss moduli were not varied significantly. This enhancement of storage moduli can be explained by the energy dissipation mechanism, resulting in more uniform distribution of load among the whole network. Under stress, polymer network 


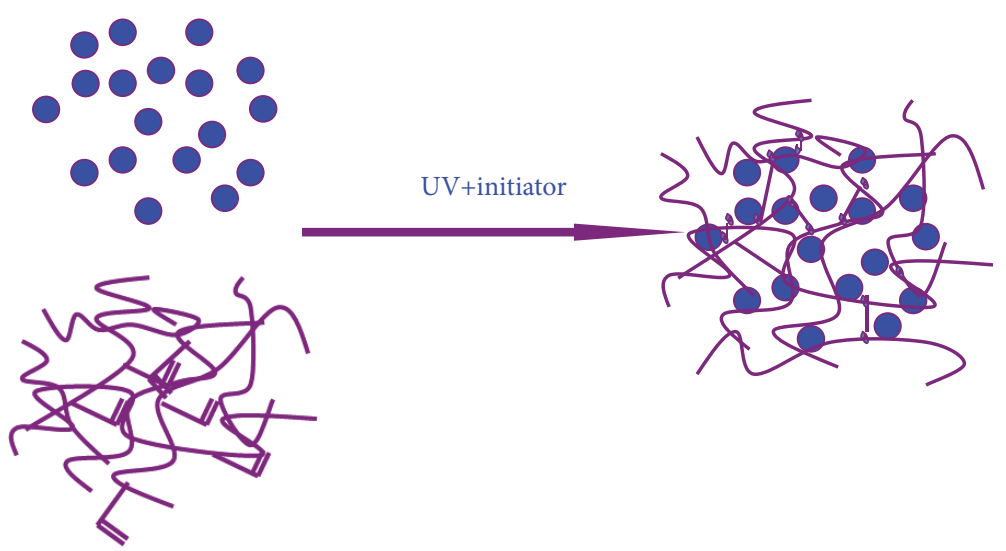

(a)

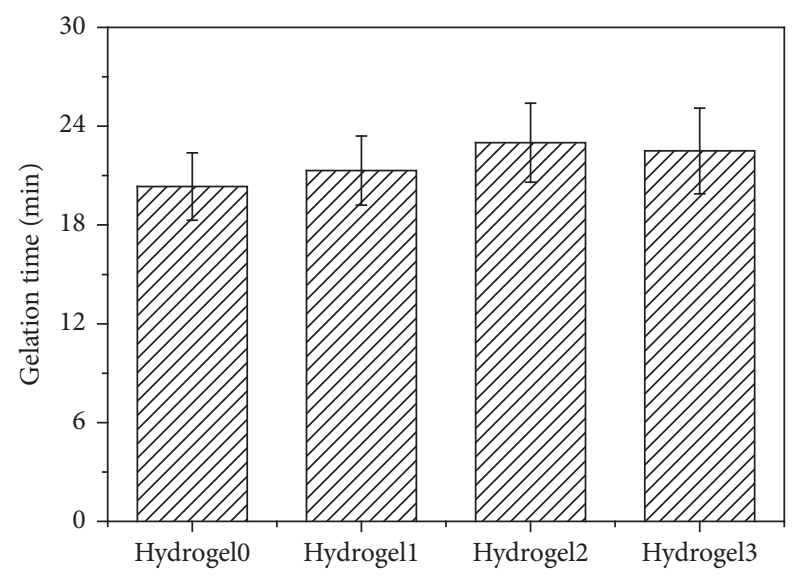

(b)

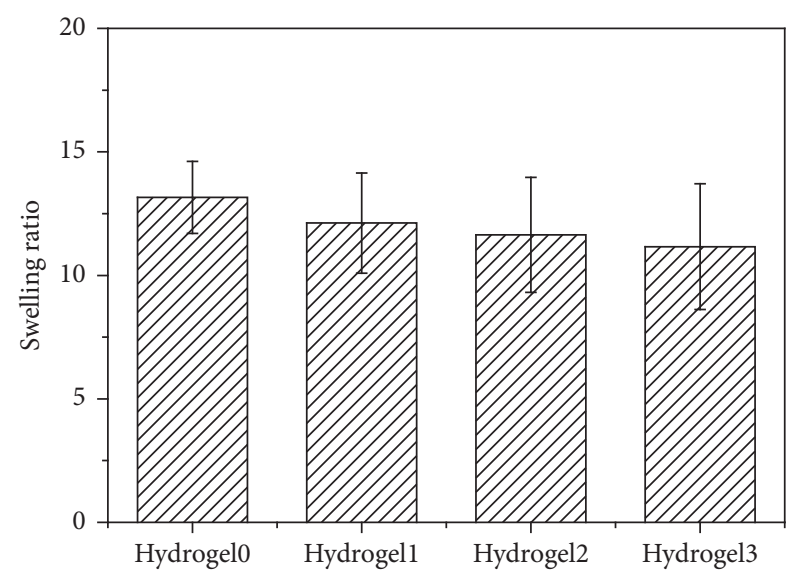

(c)

FIGURE 3: (a) Schematic illustration to show the formation of injectable hydrogel. (b) Gelation time of different hydrogels. (c) Swelling ratio of different hydrogels.

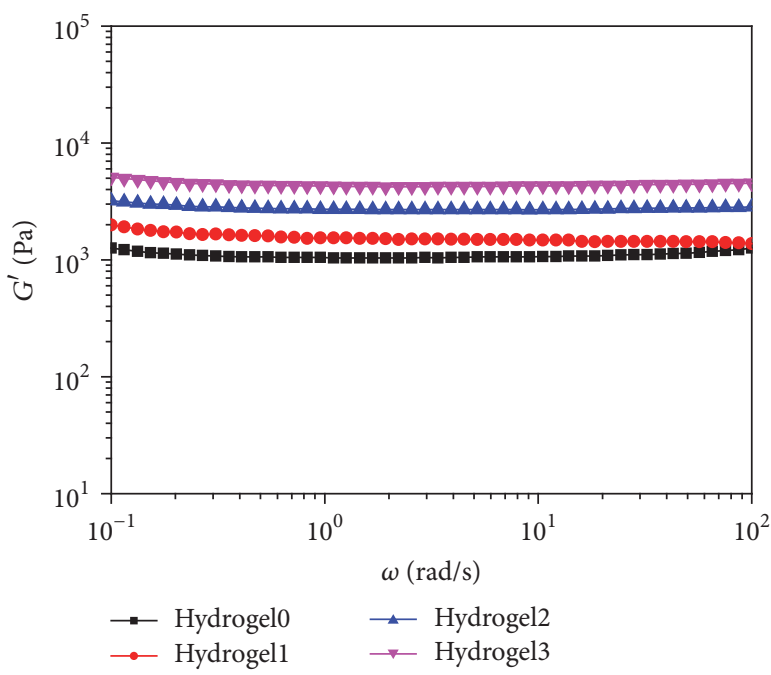

(a)

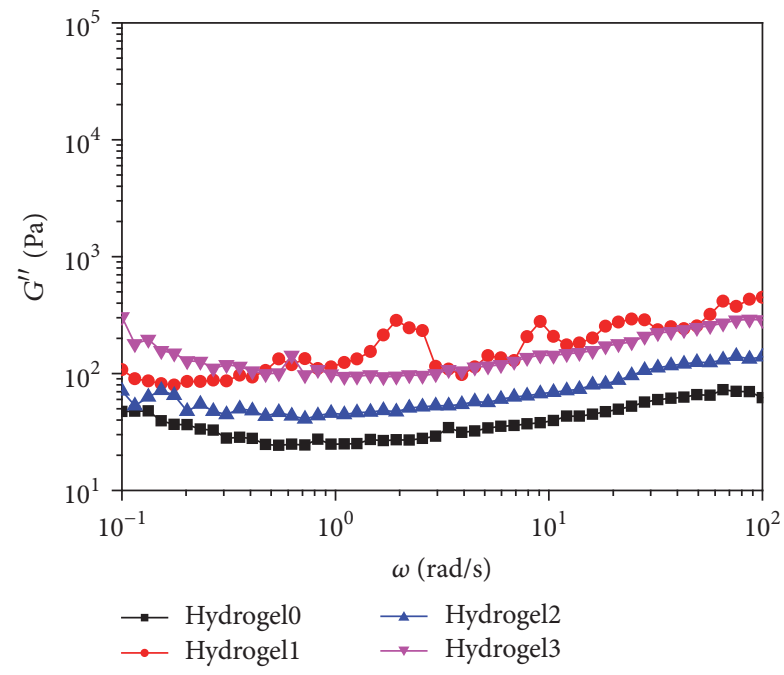

(b)

FIGURE 4: Storage modulus (a) and loss modulus (b) of different hydrogel as a function of compressing frequency $(\omega)$. Strain and temperature were set at $1 \%$ and $37^{\circ} \mathrm{C}$, respectively. 


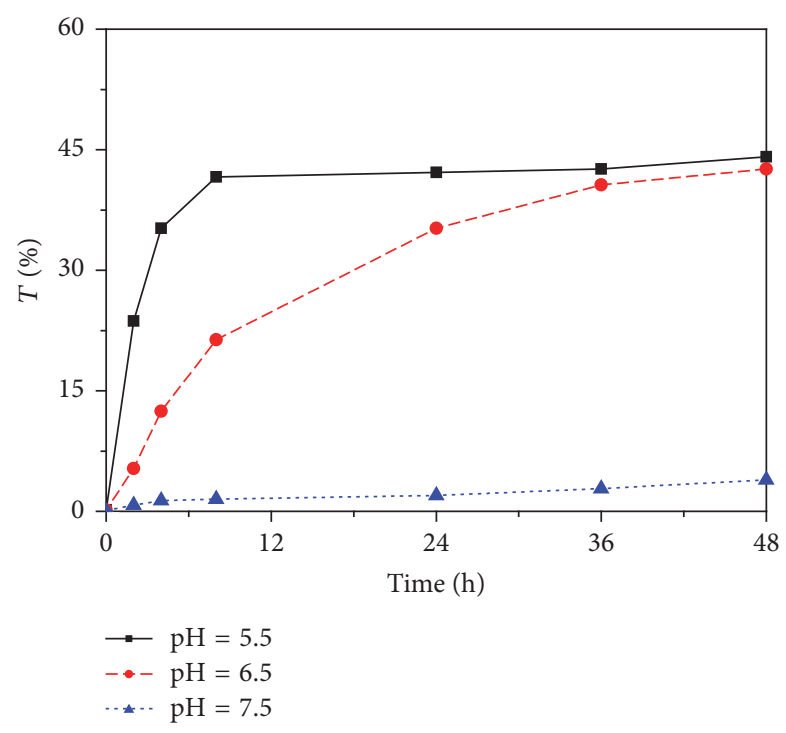

FIGURE 5: Transparency of hydrogel3 in different medium.

of hydrogel is deformed, dissipating energy to nanoparticles. Not surprisingly, more nanoparticles can endure more stress, resulting in larger storage moduli. In addition, since GAA was stably absorbed on the surface of nanoparticle in the research, the polymer chain was inevitably crosslinked with nanoparticle surface during the process of hydrogel formation, which ensured effective energy delivery under stress. When it comes to loss moduli, factors of contribution to loss moduli became viscous, which is mainly decided by the continuous phase, namely, crosslinked gelatin network of hydrogel. Therefore, no obvious trend was found for effects of nanoparticle concentration to loss moduli.

In order to evaluate the $\mathrm{pH}$ response properties, the transparency of hydrogel3 along with time in different medium was tracked in Figure 5. When pH of outer solution was 5.5, the transparency of hydrogel 3 increased quickly in first $6 \mathrm{~h}$, indicating the quick dissolution of $\mathrm{Ac}-\beta-\mathrm{CD}$ nanoparticles at acid environment. When $\mathrm{pH}$ of outer solution was 6.5, the transparency of hydrogel 3 gradually increased in $36 \mathrm{~h}$, implying relative slow degradation of Ac- $\beta$-CD nanoparticles in faintly acid environment. When $\mathrm{pH}$ of outer solution was 7.5, the transparency of hydrogel 3 increased little during the detected $48 \mathrm{~h}$, showing that the Ac- $\beta$-CD nanoparticles in hydrogel could exist stably in alkalescent environment. The transparent property of hydrogel is relevant to the degradation of nanoparticle. This $\mathrm{pH}$-controlled degradation could induce the control release of drug/bioactive factor in previous reports. In view of the above-mentioned results and analysis, it can be deduced that the composite hydrogel had obvious acid-response properties, which would broad the injectable materials in biomedical application.

\section{Conclusions}

An injectable composite hydrogel with $\mathrm{pH}$ response properties was designed and prepared for biomedical application. Firstly, double carbon functionalized gelatin (G-AA) and acetalated $\beta$-cyclodextrin (Ac- $\beta$-CD) with $\mathrm{pH}$ response property were successfully synthesized as macromonomer/ surface modifier and original material for nanoparticles, respectively. Secondly, G-AA functional Ac- $\beta$-CD nanoparticles with diameter of $238 \pm 61 \mathrm{~nm}$ were conveniently prepared by one-step emulsion method. Finally, injectable composite hydrogel was obtained by photoinitiated polymerization in situ. The gelation time and swelling ratio showed slight increase and gradual decrease along with the increase of nanoparticle concentration, respectively, but without significant difference. All investigated hydrogels in the research had typical characteristics of crosslinked elastomer. Along with the increase of nanoparticle concentration, storage moduli were augmented correspondingly, while loss moduli were not varied significantly. Moreover, the transparency with time had verified obvious acid-response properties of hydrogels.

\section{Competing Interests}

The authors declare that there are no competing interests regarding the publication of this paper.

\section{Acknowledgments}

This study is financially supported by Qing Lan Project.

\section{References}

[1] X. Hu, D. Li, H. Tan, C. Pan, and X. Chen, "Injectable graphene oxide/graphene composite supramolecular hydrogel for delivery of anti-cancer drugs," Journal of Macromolecular Science, Part A: Pure and Applied Chemistry, vol. 51, no. 4, pp. 378-384, 2014.

[2] H. Tan and K. G. Marra, "Injectable, biodegradable hydrogels for tissue engineering applications," Materials, vol. 3, no. 3, pp. 1746-1767, 2010.

[3] X. Hu, D. Li, F. Zhou, and C. Gao, "Biological hydrogel synthesized from hyaluronic acid, gelatin and chondroitin sulfate by click chemistry," Acta Biomaterialia, vol. 7, no. 4, pp. 1618-1626, 2011.

[4] J. Chen, X. Chen, X. Yang, C. Han, C. Gao, and Z. Gou, "Bioactive glasses-incorporated, core-shell-structured polypeptide/ polysaccharide nanofibrous hydrogels," Carbohydrate Polymers, vol. 92, no. 1, pp. 612-620, 2013.

[5] S. T. Koshy, R. M. Desai, P. Joly et al., "Click-crosslinked injectable gelatin hydrogels," Advanced Healthcare Materials, vol. 5, no. 5, pp. 541-547, 2016.

[6] Z. Li, T. Qu, C. Ding et al., "Injectable gelatin derivative hydrogels with sustained vascular endothelial growth factor release for induced angiogenesis," Acta Biomaterialia, vol. 13, pp. 88-100, 2015.

[7] B. Qi, A. Yu, S. Zhu, B. Chen, and Y. Li, "The preparation and cytocompatibility of injectable thermosensitive chitosan/poly(vinyl alcohol) hydrogel," Journal of Huazhong University of Science and Technology [Medical Sciences], vol. 30, no. 1, pp. 89-93, 2010.

[8] K. Wang, K. C. Nune, and R. D. K. Misra, "The functional response of alginate-gelatin-nanocrystalline cellulose injectable hydrogels toward delivery of cells and bioactive molecules," Acta Biomaterialia, vol. 36, pp. 143-151, 2016. 
[9] X. Hu, J. Zhou, N. Zhang, H. Tan, and C. Gao, "Preparation and properties of an injectable scaffold of poly(lactic-co-glycolic acid) microparticles/chitosan hydrogel," Journal of the Mechanical Behavior of Biomedical Materials, vol. 1, no. 4, pp. 352-359, 2008.

[10] Q. X. Ji, X. G. Chen, Q. S. Zhao, C. S. Liu, X. J. Cheng, and L. C. Wang, "Injectable thermosensitive hydrogel based on chitosan and quaternized chitosan and the biomedical properties," Journal of Materials Science: Materials in Medicine, vol. 20, no. 8, pp. 1603-1610, 2009.

[11] J. Sun, G. Jiang, T. Qiu, Y. Wang, K. Zhang, and F. Ding, "Injectable chitosan-based hydrogel for implantable drug delivery: body response and induced variations of structure and composition," Journal of Biomedical Materials Research-Part A, vol. 95, no. 4, pp. 1019-1027, 2010.

[12] M. Busso and R. Voigts, "An investigation of changes in physical properties of injectable calcium hydroxylapatite in a carrier gel when mixed with lidocaine and with lidocaine/epinephrine," Dermatologic Surgery, vol. 34, no. 1, pp. S16-S24, 2008.

[13] S. Herberg, M. Siedler, S. Pippig et al., "Development of an injectable composite as a carrier for growth factor-enhanced periodontal regeneration," Journal of Clinical Periodontology, vol. 35, no. 11, pp. 976-984, 2008.

[14] X. G. Zhang, D. Y. Teng, Z. M. Wu et al., "PEG-grafted chitosan nanoparticles as an injectable carrier for sustained protein release," Journal of Materials Science: Materials in Medicine, vol. 19, no. 12, pp. 3525-3533, 2008.

[15] S. M. Lim, H. J. Lee, S. H. Oh, J. M. Kim, and J. H. Lee, "Novel fabrication of PCL porous beads for use as an injectable cell carrier system," Journal of Biomedical Materials Research-Part B Applied Biomaterials, vol. 90, no. 2, pp. 521-530, 2009.

[16] Y. Ye and X. Hu, "A pH-sensitive injectable nanoparticle composite hydrogel for anticancer drug delivery," Journal of Nanomaterials, vol. 2016, Article ID 9816461, 8 pages, 2016.

[17] Q. Shi, L. Zhang, M. Liu et al., "Reversion of multidrug resistance by a pH-responsive cyclodextrin-derived nanomedicine in drug resistant cancer cells," Biomaterials, vol. 67, pp. 169-182, 2015.

[18] Z. Zhou, A. Badkas, M. Stevenson, J.-Y. Lee, and Y.-K. Leung, "Herceptin conjugated PLGA-PHis-PEG pH sensitive nanoparticles for targeted and controlled drug delivery," International Journal of Pharmaceutics, vol. 487, no. 1-2, pp. 81-90, 2015.

[19] H. M. Borteh, M. D. Gallovic, S. Sharma et al., "Electrospun acetalated dextran scaffolds for temporal release of therapeutics," Langmuir, vol. 29, no. 25, pp. 7957-7965, 2013.

[20] H. Chen, X. Liu, Y. Dou et al., "A pH-responsive cyclodextrinbased hybrid nanosystem as a nonviral vector for gene delivery," Biomaterials, vol. 34, no. 16, pp. 4159-4172, 2013.

[21] H. He, S. Chen, J. Zhou et al., "Cyclodextrin-derived pHresponsive nanoparticles for delivery of paclitaxel," Biomaterials, vol. 34, no. 21, pp. 5344-5358, 2013.

[22] S. Suarez, G. N. Grover, R. L. Braden, K. L. Christman, and A. Almutairi, "Tunable protein release from acetalated dextran microparticles: a platform for delivery of protein therapeutics to the heart post-MI," Biomacromolecules, vol. 14, no. 11, pp. 39273935, 2013. 

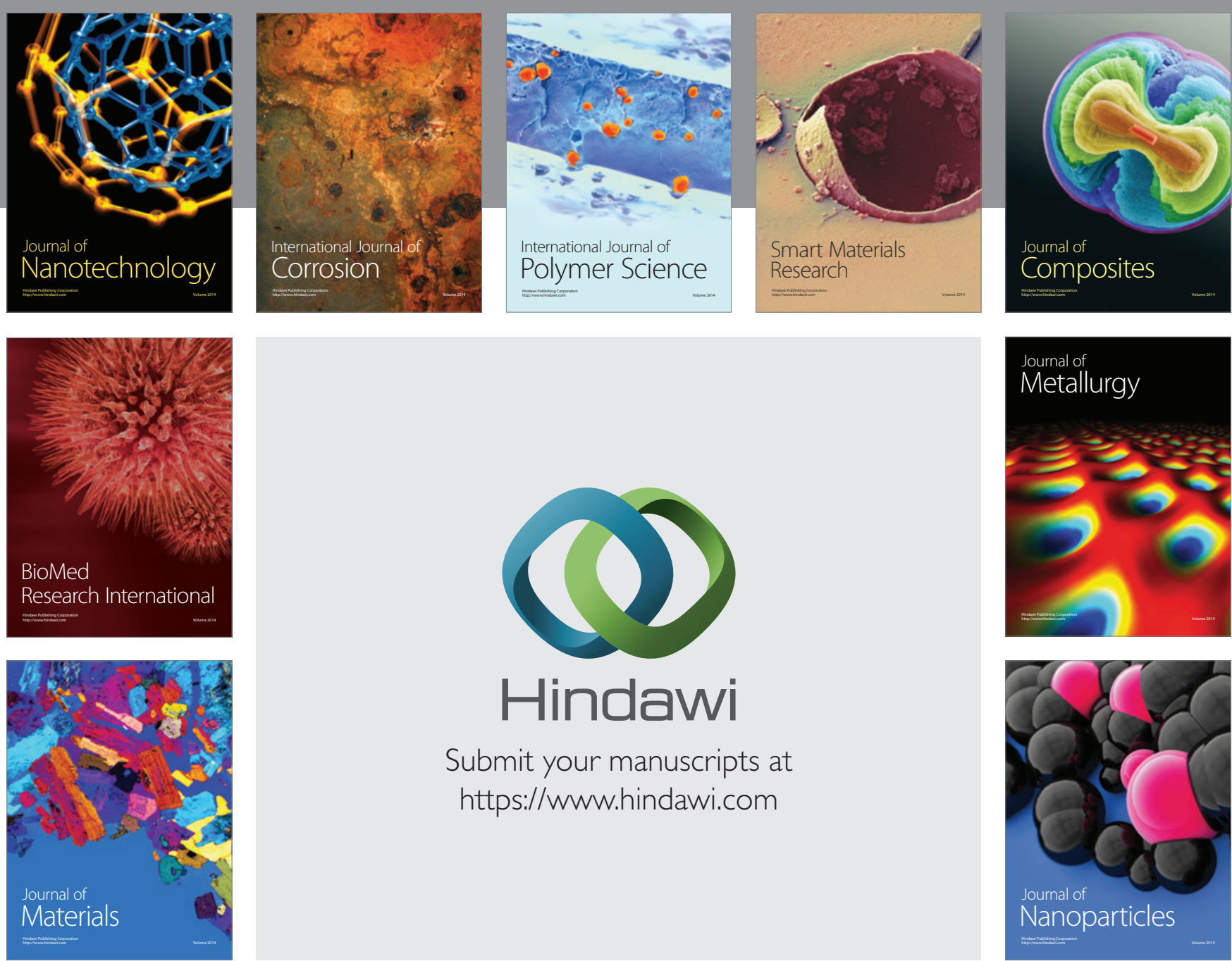

\section{Hindawi}

Submit your manuscripts at

https://www.hindawi.com

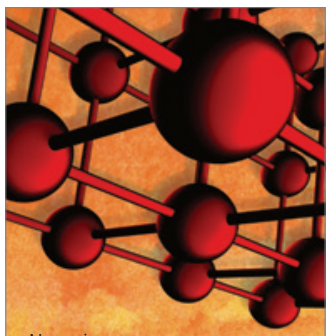

Materials Science and Engineering
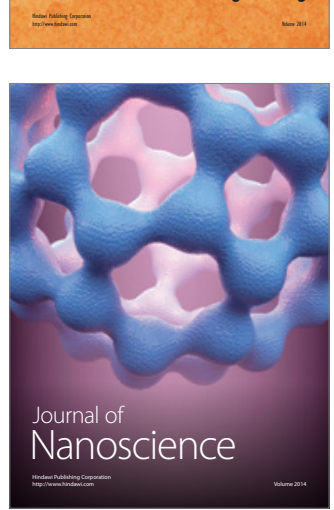
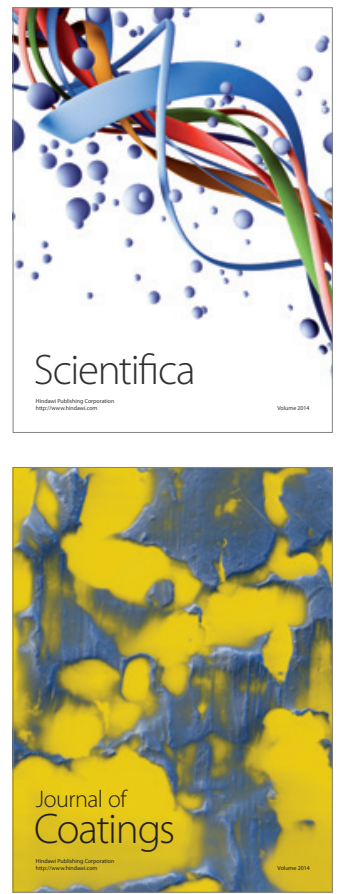
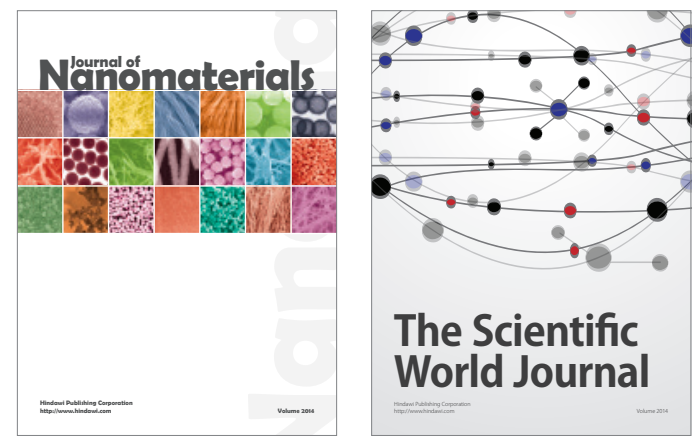

The Scientific World Journal
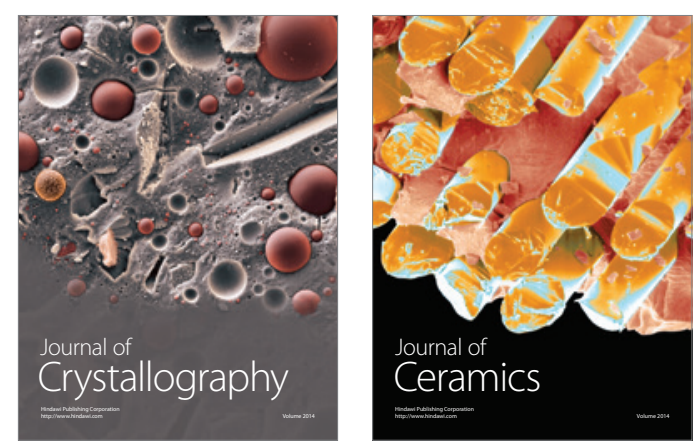
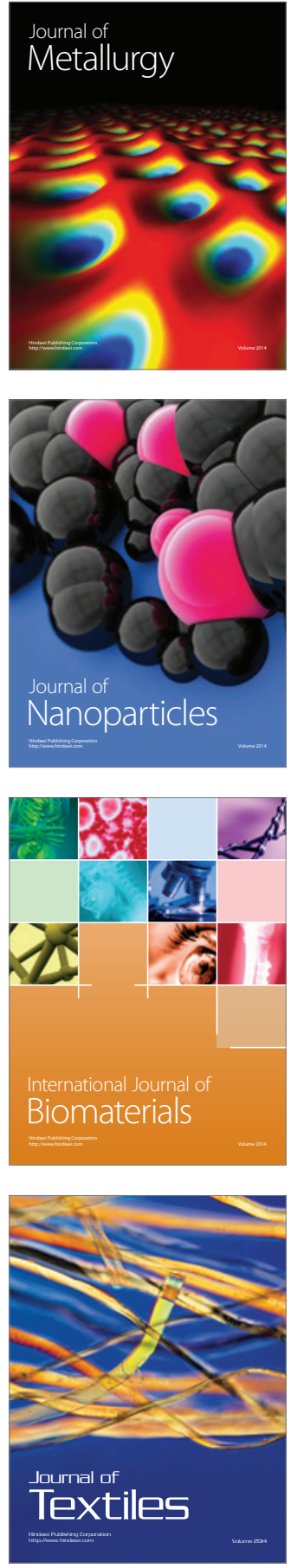